Lekhooaetal, Afr J Tradit Complement Altern Med. (2012) 9(3S):73-80

\title{
EVALUATION OF TRADITIONAL MEDICINES II: THE USE OF METABOLITE PEAK-KINETICS TO MONITOR PHELA IN RAT PLASMA.
}

\section{Makhotso Lekhooa*, Andrew Walubo*, Jan JB Du Plessis*, and Motlalepula G. Matsabisa ${ }^{\# 1}$}

\author{
*University of the Free State, Department of Pharmacology \\ ${ }^{1}$ Medical Research Council, Indigenous Knowledge Systems (Health) Unit \\ P.O. Box 19070, Tygerberg 7505, Francie van Zijl Drive Parow Valley, Cape Town, South Africa
}

\#E-mail: motlalepula.matsabisa@mrc.ac.za

\begin{abstract}
PHELA is a herbal mixture of four African traditional medicinal plants that is under development by the Medical Research Council (MRC) for use as an immune stimulant in immune compromised individuals. Before major in vivo investigations could be conducted, there was a need to establish a plasma marker for concentration monitoring of PHELA. Chromatographic separation was achieved using a $\mathrm{C}_{18} \mathrm{RP}$ column (250 $\left.\mathrm{mm} \times 4.6 \mathrm{~mm} \times 5 \mu \mathrm{m}\right), 70 \%$ acetonitrile in water and fluorescent detection. Three groups of rats $(n=5)$ were administered with PHELA $(15.4 \mathrm{mg} / \mathrm{kg})$ and one rat from each group was sacrificed at 1,2 , 4, 6 and 8 hours. Surprisingly, on the HPLC analysis, none of the marker peaks of spiked plasma were detectable in the plasma of treated animals. Instead, a new peak was observed at 9.2 minutes, which implied that it was a metabolite of PHELA. Using peak area per unit plasma volume (PK-area/L), the relevant pharmacokinetic parameters were derived. The metabolite's half-life was $3.47 \pm 0.35$ hours and reached maximum concentration at $4.67 \pm 1.15$ hrs. It was estimated that with once daily dosing of PHELA, the concentration at steady state (Css) would be $47.52 \pm 5.94$ PK-area/L with no drug accumulation (Acc index $=.009 \pm 0.004$ ). In conclusion, the use of peak area per unit volume to derive pharmacokinetics of unknown compounds (Peak-kinetics) and to confirm ingestion of PHELA were demonstrated with a hope that they may appeal to those experiencing similar problems with monitoring of herbal products of which little is known.
\end{abstract}

Key words: PHELA, Herbal medicine, Peak-kinetics, plasma, HPLC method

\section{Introduction}

Owing to the inaccessibility and inadequacy of existing Western medicines, the use of traditional medicines is increasingly gaining importance. Already, the World Health Organization (WHO) and many governments and other non-government organizations are championing the development of cheaper and effective medicines for neglected diseases from traditional medicines, and this has been paralleled by calls to ensure the safety of traditional medicines [(WHO, 2000; Government Gazette, 2008; Peschel, 2007). One such approach has been to undertake concentration monitoring using known compounds in the product as plasma markers (Jacobson et al, 2001; Drago et al, 2002; Kuge et al, 2003). Plasma markers are useful in the concentration monitoring of products in order to determine, to mention but a few, proof of ingestion and absorption of the product, change in dose, potential host problems, therapeutic end points, toxicity and in evaluating for variations in response among individuals. Therefore, it is important to establish a plasma marker for any traditional medicines in development.

PHELA is a herbal mixture of four African traditional medicinal plants [Clerodendrum glabrum, Polianthes tuberosa, Rotheca myricoides and Senna occidentails] that is under development by the Medical Research Council (MRC) for use as an immune stimulant in immune compromised individuals. Before in vivo investigations could be done, there was a need to establish a plasma marker for concentration monitoring of PHELA. The plasma marker is important for setting the pharmacokinetic parameters (conditions) under which results of the subsequent studies would be obtained to ensure reproducibility of data. Furthermore, the pharmacokinetic parameters will be used in establishing the appropriate dose and dosing frequency for PHELA.

Unfortunately, concentration monitoring of traditional medicines is not applicable to most products owing, not only to the complex nature of the products, but also due to lack of knowledge of the active or toxic ingredients by which to monitor the concentrations or therapeutic response or toxicity. Furthermore, the individual compounds are in low concentrations such that they may not be detected or targeted by the current analytical methods. On the other hand, therapeutic activity may be due to multiple compounds whereby one compound, the so called marker compound, may not correlate with response or toxicity. Even then, concentration monitoring requires development of a method for analysis of the marker compound, and such a method has to be validated to ensure robustness before use. This is a challenge for traditional medicines because there is no guarantee that the compounds or chromatographic peaks observed in the product shall be seen in plasma after administration of the product. Furthermore, given that the compounds detected are unknown, and therefore the dose and other factors are unknown too, it creates another challenge in the use of conventional units such as milligrams per liter (mg/L) for plasma concentrations.

Therefore, there is a need for innovative techniques by which to ensure safety of traditional medicines. Specifically, the current approach to concentration monitoring and assay method development and/or selection of marker compounds is not conducive to evaluation of unknown compounds in traditional medicines for use as plasma markers. There is a need for a paradigm shift in the manner we undertake evaluation and safety monitoring of 
medicines. Here, we describe the development of a high performance liquid chromatography (HPLC) method for monitoring PHELA in plasma using the peak of unknown compound as a plasma marker, and the subsequent use of its peak-area for pharmacokinetic analysis (Peak-kinetics), with a hope that it will open avenues for innovation in the safety evaluation of traditional medicines in general.

\section{Materials and Methods Apparatuses}

A vortex mixer (Scientific Industries Inc, U.S.A) was used for mixing. A 5810 R centrifuge and mini spin (Eppendorf, Germany) were used for centrifuging and quick spinning of samples. Precision balances SBC 31 and SPB 52 (Scaltec Instruments, Germany) were used for weighing milligram and gram quantities respectively. A nitrogen evaporator with a heating block was used for evaporating samples. Finnpipettes from Thermo Labsystems (Canada) were used for spiking volumes. Glass pipette attached was used for adding extraction solvents

\section{Chemicals and reagents}

PHELA was manufactured and supplied by the Indigenous Knowledge Unit (IKS) of the South African Medical Research Council. HPLC grade solvents: hexane, methanol, acetonitrile, diethyl ether, toluene (Burdick and Jackson, U.S.A) and hydrochloric acid, (Merck, Germany) were used during sample preparation. Sodium hydroxide and ammonia sulphate were purchased from Sigma -Aldrich (Germany). Deionised and distilled water was prepared by Millipore water system (Milli -Q ${ }^{T m}$ ).

\section{HPLC system and conditions}

The HPLC system was an HP (Hewelt-Parkard) 1100 series consisting of an isocratic pump, autosampler and programmable fluorescent detector (HP-1046A; Germany) with emission and excitation wavelengths set at $210 \mathrm{~nm}$ and $290 \mathrm{~nm}$, respectively, connected to a computer with LCChemStation software. The sample was eluted with $70 \%$ of acetonitrile in water $(70: 30 ; \mathrm{v} / \mathrm{v})$ and separated on a reverse phase $\mathrm{C}_{18}(250 \mathrm{~mm} \times 4.6 \mathrm{~mm} \times$ $5 \mu \mathrm{m}$ ) column coupled to a $\mathrm{C}_{18}$ guard column at a flow rate of $0.5 \mathrm{ml} / \mathrm{min}$.

\section{Sample preparation}

To $0.5 \mathrm{ml}$ of plasma was added $100 \mathrm{mg}$ ammonia sulphate $\left\{\left(\mathrm{NH}_{4}\right)_{2} \mathrm{SO}_{4}\right\}$ after which Iso-propanol $(1 \mathrm{ml})$ was added and shaken for 10 minutes. Thereafter, it was centrifuged at $4{ }^{\circ} \mathrm{C}$ and $2500 \mathrm{rpm}(1251 \mathrm{~g})$ for 10 minutes. The organic layer was evaporated under nitrogen at $47^{\circ} \mathrm{C}$, and residues reconstituted with $100 \mu$ l of mobile phase and $50 \mu$ I was injected in to the HPLC.

\section{Standardization}

The retention times for marker peaks in spiked plasma was standardized as mean \pm s.d. by running spiked sample over six days $(n=6)$. However, validation was not done owing to the lessons learned from preliminary HPLC-UV experiments where after going through all the validation, none of the peaks observed in spiked plasma were detectable in plasma from treated animals. Therefore, plasma samples from treated rats were extracted and analyzed early in HPLC-FLD method development.

\section{Animal Experiment}

The study was approved by the animals Ethics committee of the University of the Free State. Fifteen male Sprague Dawley rats were divided into three groups of 5 animals each. All the animals were then administered orally with an aqueous solution of PHELA at a dose of $15.4 \mathrm{mg} / \mathrm{kg}$. Thereafter, one animal from each group was sacrificed after 1, 2, 4, 6 and 8 hours of dosing. Under ether anesthesia, blood samples were collected by cardiac puncture in a heparin tube. The samples were placed on ice, after which they were centrifuged and plasma separated within 30 minutes. Plasma was stored at $-20^{\circ} \mathrm{C}$ until analysis. 
For evaluation of the metabolite kinetics, the concentration of the metabolite was expressed as the metabolite's peak area (PK-area) per unit plasma volume (L), by dividing the peak area by sample volume (i.e., PK-area/L). This concentration was then used to derive the necessary pharmacokinetic parameters (i.e., Peak-kinetics; defined as "the use of peaks in plasma chromatograms to determine the pharmacokinetics of a compound"). In effect, the pharmacokinetics formulas remain the same.

Pharmacokinetic parameters of the marker peak for each group of animals were obtained from plasma concentration (PK-area/ml) versus time plots by non-compartmental methods, whereby the area under plasma concentrations versus time (AUC) were calculated using the trapezoidal rule with extrapolation to infinity (Ct/Ke) (Gibaldi et. al., 1984). The elimination rate constant (Ke) for the decline of plasma concentrations was obtained by linear regression on log plasma concentration against time plots. Thereafter, the following equations were used to derive the relevant pharmacokinetic parameters:

Maximum concentration (Cmax) and time to reach $\mathrm{Cmax}(\mathrm{Tmax})$ were read of directly from the concentration time curve.

- Half-life $\left(T_{1 / 2(m)}\right)=\frac{0.693}{K e_{(m)}}$

- $\quad$ Mean Residence Time $\left(\mathrm{MRT}_{(\mathrm{m})}\right)=\frac{1}{K e_{(m)}}$

Prediction of concentration of metabolite at steady state $\left(\mathrm{C}_{\mathrm{ss}(\mathrm{m})}\right)$ at specific dosing interval $(\tau)$ and the extent of drug accumulation (Accumulation index; Accindex) on multiple dosing were calculated as follows:

- Conc. of metabolite at steady state $\left(\mathrm{C}_{\mathrm{ss}(\mathrm{m})}\right)=\frac{A U C_{m}}{\tau}$

- Accumulation index $(\operatorname{Accindex}(m))=\frac{1}{\left(1-e^{-k \tau}\right)}$

Eq. 4

\section{Statistical data analysis:}

Data was analyzed by non-parametric methods using the Graph Pad statistical program. Accordingly, parameters were reported as median and range, and the Mann Whitney Test was used for data comparison with the level of significance set at $p<0.05$. 
RESULTS

\section{Chromatogram Performance}

Figure 1 shows the representative HPLC-FLD chromatogram for blank plasma (A), spiked plasma (B) and rat plasma (C) six hours after oral administration of PHELA. The spiked plasma shows markers peaks at 12,22 and 32 minutes which was within the standardized range of $12.2 \pm 0.28$ minutes for peak (a), $22.3 \pm 1.18$ minutes for peak (b) and $32.96 \pm 0.88$ minutes for peak $(c)(n=6)$. There were no marker peaks in the plasma of rats administered with PHELA, but there was a new peak at 9.22 minutes. The peak size increased with time to a maximum and then fell off, typical of time versus concentration curve, i.e., the peak areas (\% $\left.\mathrm{F}^{\star} \mathrm{S}\right)$ were 116 at 1 hour, 156 at 2 hours, 204 at 4 hours, 156 at 6 hours and 60 at 8 hours after oral administration of PHELA (Figure 2). Since this peak was not observed in the spiked plasma, it implied that it was a metabolite of PHELA. Therefore, it was decided to use this peak as a marker for monitoring of PHELA in plasma.
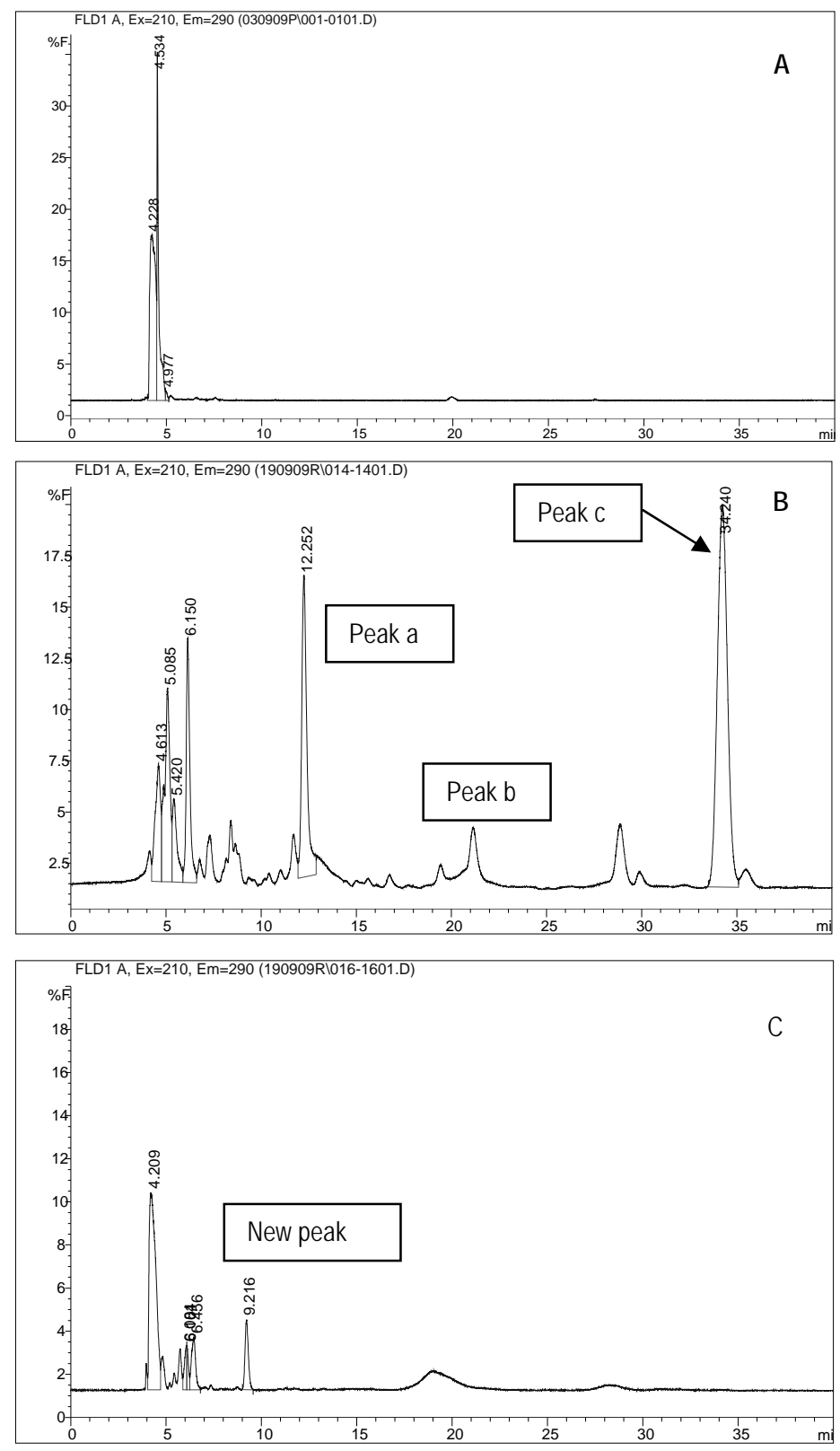

Figure 1: Representative HPLC-FLD chromatogram of blank plasma (A), spiked plasma (B) and rat plasma (C) six hours after oral administration of PHELA.

http://dx.doi.org/10.4314/ajtcam.v9i3S.9 

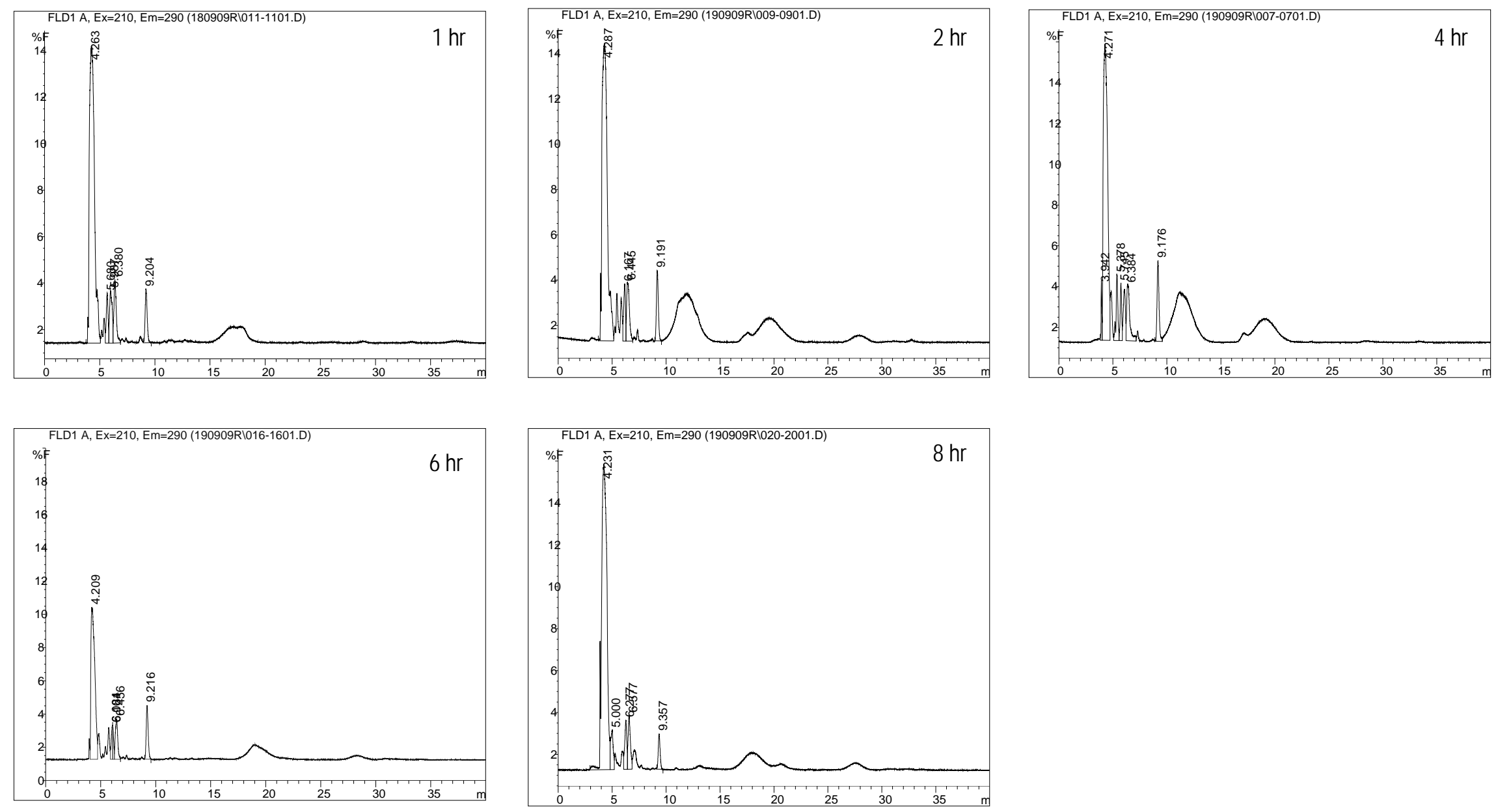

Figure 2: HPLC-FL chromatograms illustrating the changes in peak area of RT 9.19-9.3 minutes, after 1 hour (58 peak area/ml), 2 hours (78 peak area/ml), 4 hours (102 peak area/ml), 6 hours (78 peak area/ml) and 8 hours (30 peak area/ml) of oral administration of PHELA. 


\section{Metabolite Peak-kinetics}

Figure 3 is the concentration versus time plot for PHELA's metabolite in rats after oral administration, while Table 1 shows the pharmacokinetic parameters of the metabolite derived from the plasma concentration profile in the same animals. The animals weights were within the same range and so was the dose. Except for the Tmax of 6 hours in group 2, the rest of the kinetic parameters were similar in the three groups. Owing to lack of knowledge on the parent compound kinetic parameters, it was not possible to determine whether the metabolite's rate limiting elimination step is in the formation or elimination process.

The predictive parameters showed that concentration of the metabolite at steady state would be approximately $48 \mathrm{PK}$-areas $/ \mathrm{ml}$, and that this concentration at steady state will be similar to that observed after single dose administration (Acc. index $=1$ ), which implies that there will be no accumulation of the drug. Since the dose of metabolite is unknown, and urine was not collected, it was not possible to calculate clearance and volume distribution of the metabolite.

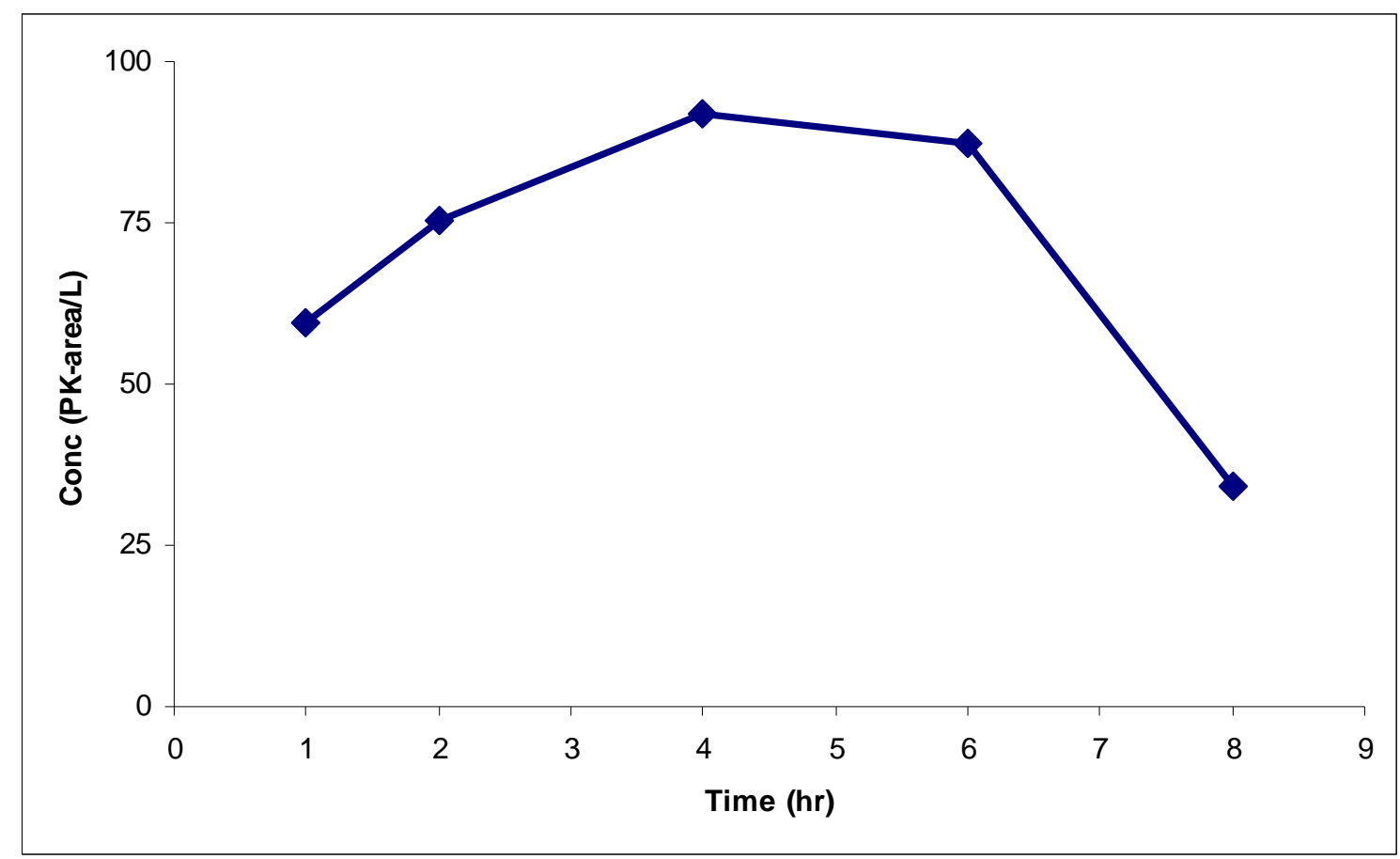

Figure 3: The average plasma concentrations of PHELA's metabolite in rats $(n=3)$ after oral administration of PHELA. 
Lekhooaetal, Afr J Tradit Complement Altern Med. (2012) 9(3S):73-80

Table 1: Plasma pharmacokinetic parameters of PHELA's metabolite in rats after oral administration of PHELA.

\begin{tabular}{|c|c|c|c|c|c|c|}
\hline & Group 1 & Group 2 & Group 3 & Mean & \pm & S.D. \\
\hline Wt (kg) & 0.377 & 0.349 & 0.389 & \multicolumn{3}{|c|}{$0.372 \pm 0.02$} \\
\hline Dose (mg) & 5.80 & 5.37 & 6.00 & \multicolumn{3}{|c|}{$5.72 \pm 0.32$} \\
\hline $\mathrm{Co}_{\mathrm{m}}$ (Pk-area/L) & 270.05 & 231.47 & 187.91 & \multicolumn{3}{|c|}{$229.81 \pm 41.10$} \\
\hline $\mathrm{Ke}_{\mathrm{m}}(/ \mathrm{hr})$ & 0.207 & 0.216 & 0.180 & \multicolumn{3}{|c|}{$0.201 \pm 0.02$} \\
\hline Half-life $_{m}(\mathrm{hr})$ & 3.35 & 3.20 & 3.85 & \multicolumn{3}{|c|}{$3.47 \pm 0.35$} \\
\hline AUC $_{m}($ PK-area.hr/L) & 1304.47 & 1070.19 & 1045.90 & \multicolumn{3}{|c|}{$1140.52 \pm 142.45$} \\
\hline $\mathbf{M R T}_{\mathrm{m}}(\mathrm{hr})$ & 4.83 & 4.62 & 5.57 & \multicolumn{3}{|c|}{$5.01 \pm 0.50$} \\
\hline$F_{m}(\%)$ & 1 & 1 & 1 & \multicolumn{3}{|l|}{$1 \pm 0$} \\
\hline $\operatorname{Tmax}_{m}(\mathrm{hr})$ & 4 & 6 & 4 & \multicolumn{3}{|c|}{$4.67 \pm 1.15$} \\
\hline $\operatorname{Cmax}_{m}($ Pk-area/L) & 118 & 104 & 82 & \multicolumn{3}{|c|}{$101.33 \pm 18.15$} \\
\hline \multicolumn{7}{|l|}{ Predictive parameters } \\
\hline $\mathrm{C}_{\mathrm{ss}(\mathrm{m})} \quad(\mathrm{Pk}$-area/L) & 54.35 & 44.59 & 43.62 & 47.52 & \pm & 5.94 \\
\hline $\operatorname{Accc}_{\text {Index(m) }}$ & 1.007 & 1.006 & 1.014 & \multicolumn{3}{|c|}{$1.009 \pm 0.004$} \\
\hline
\end{tabular}

Key: $\mathbf{A U C}_{\mathrm{m}}=$ Area under the plasma concentration-time curve for the metabolite; $\mathrm{CLp}_{\mathrm{m}}=$ plasma clearance of the metabolite; $\mathbf{C m a x}_{\mathrm{m}}=$ maximum concentration; $\mathrm{Co}_{\mathrm{m}}=$ concentration of the metabolite after instantaneous distribution at time zero; $\mathbf{F}=$ bioavailability of the metabolite; $\mathrm{Ke}=$ elimination

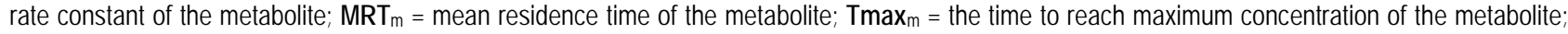
$\mathbf{V} \mathbf{d}_{\mathrm{m}}=$ volume of distribution of the metabolite; and $\mathbf{W t}=$ weight

\section{Discussion}

Here, a method for monitoring PHELA in rat plasma by HPLC-FLD has been described. The method was used to analyze PHELA's metabolite in plasma which was then utilized as the plasma marker for PHELA. Since the metabolite is unknown there was no standard by which to express its plasma concentration in conventional units and the changes in peak area per unit volume of plasma $(L)$ were used to derive the appropriate pharmacokinetic parameters, hence the term Peak-kinetics. Please note, Peak-kinetics is a process, while the derived parameters are true pharmacokinetic parameters of the unknown compound.

On the other hand, the failure to detect marker peaks seen in spiked plasma was a clear indication that the orthodox approach of developing and validating a method for analysis of pure compound before its application to in vivo samples does not apply to herbal medicines or complex mixtures. As a result, some innovation was effected by testing samples of treated animals before validation could be attempted. In fact, this advance testing obviated the validation process because a metabolite was found instead. It is therefore important that everybody, researchers and ethics committees, consider in good light, applications for administration of herbal or traditional medicines to subjects for analytical method development.

The peak area was successfully used to derive the appropriate pharmacokinetic parameters. There is no doubt that the pharmacokinetic parameters obtained will be the same even after purifying the metabolite and converting to the appropriate conventional concentration units. This enabled the setting of experimental conditions, i.e., that when animals are given a single dose of $P H E L A$ of $15.4 \mathrm{mg} / \mathrm{kg}$, similar pharmacokinetic parameters to those in Table 1 shall be obtained, and that all associated therapeutic or toxic response shall require these same conditions to be reproduced. It was also established by predictive kinetics that at the dose of $15.4 \mathrm{mg}$ once daily, a steady state concentration of $48 \mathrm{PK}$-area/ml would be obtained, and that there would be no accumulation of the drug. Please note the formulas enable the dosage to be varied until a desired plasma concentration at steady state is achieved. In the current study, the derived dosing schedule of $15.4 \mathrm{mg} / \mathrm{kg} /$ day shall be used in the subsequent experiments to investigate the mechanism of action of PHELA. 
In conclusion, the use of peak area to derive pharmacokinetics of unknown compounds as well as to monitor PHELA, a herbal product, was demonstrated with a hope that they may be useful to those experiencing similar problems with monitoring of products of which little is known.

\section{REFERENCES}

1. Drago F, Floriddia ML, Cro M and Giuffrida S (2002). Pharmacokinetics and bioavailability of a Ginkgo biloba extract. J. Ocular Pharmacol. Ther., 18: 197-202.

2. Gibaldi, M., (1984). Non-compartmental pharmacokinetics. In: M. Gibaldi, (ed.), Biopharmaceutics and Clinical Pharmacokinetics. Philadelphia: Lea and Febiger, pp. 17-28.

3. Government Gazette, (2008). African Traditional Medicine Draft Policy for South Africa Effective on 25 Jul 2008. Available at: http://www.pmg.org.za/files/docs/080725health-traditionalmedicinepolicy.pdf [Accessed on 23rd December 2009]

4. Jacobson, J. M., Feinman, L., Liebes, L., Ostrow, N., Koslowski, V., Tobia, A., Cabana, B. E., Lee, D.-H., Spritzler, J., Prince, A. M., (2001). Pharmacokinetics, safety, and antiviral effects of hypericin, a derivative of St. John's wort plant, in patients with chronic hepatitis $\mathrm{C}$ virus infection. Antimicr. Agents Chemother., 45: 517-524.

5. Kuge T, Shibata T, and Willett MS, (2003). Wood creosote, the principal active ingredient of seirogan, an herbal antidiarrheal medicine: a single-dose, dose-escalation safety and pharmacokinetic study. Pharmacotherapy, 23: 1391-1400.

6. Peschel W,(2007). The Traditional Herbal Medicine Directive within the European regulatory framework for Herbal Products. Boletin latinoamericano y del caribe de plantas medicinales y aromaticas, 6: $102-111$. Available at: http://www.uv.es/prietojm/BLACPMA/ArchivoSep/Peschel a BLACPMA V6 N4.pdf [Accessed on 23rd December 2009]

7. WHO (2002). General Guidelines for Methodologies on Research and Evaluation of Traditional Medicines, Geneva, p. 1. 